This document is confidential and is proprietary to the American Chemical Society and its authors. Do not copy or disclose without written permission. If you have received this item in error, notify the sender and delete all copies.

\title{
High-yield synthesis of transglycosylated mogrosides improves the flavor profile of monk fruit extract sweeteners
}

\begin{tabular}{|r|l|}
\hline Journal: & Journal of Agricultural and Food Chemistry \\
\hline Manuscript ID & jf-2020-072674.R1 \\
\hline Manuscript Type: & Article \\
\hline Dute Submitted by the & n/a \\
\hline Complete List of Authors: & $\begin{array}{l}\text { Muñoz-Labrador, Ana; Instituto de Investigación en Ciencias de la } \\
\text { Alimentación CIAL (CSIC-UAM), Bioactividad y Análisis de Alimentos } \\
\text { Azcarate, Silvana; Universidad Nacional de La Pampa, Chemistry; } \\
\text { Consejo Nacional de Investigaciones Científicas y Técnicas (CONICET) } \\
\text { Lebrón-Aguilar, Rosa; Instituto de Quimica Fisica Rocasolano, } \\
\text { Quintanilla-López, Jesús Eduardo; Instituto de Química Física } \\
\text { Rocasolano, } \\
\text { Galindo-Iranzo, Placido; Instituto de Quimica Fisica Rocasolano } \\
\text { Kolida, Sofia; OptiBiotix Health plc } \\
\text { Methven, Lisa; University of Reading, Food Biosciences } \\
\text { Rastall, Robert; University of Reading, Food Biosciences } \\
\text { Moreno, F Javier; Instituto de Investigación en Ciencias de la } \\
\text { Alimentación CIAL (CSIC-UAM), Bioactividad y Análisis de Alimentos } \\
\text { Hernandez-Hernandez, Oswaldo; Institute of Food Science Research, }\end{array}$ \\
\hline
\end{tabular}

\section{SCHOLARONE \\ Manuscripts}




\section{High-yield Synthesis of Transglycosylated}

\section{Mogrosides Improves the Flavor Profile of Monk}

\section{Fruit Extract Sweeteners}

Ana Muñoz-Labrador ${ }^{1}$, Silvana Azcarate², Rosa Lebrón-Aguilar³ , Jesús

E. Quintanilla-López ${ }^{3}$, Plácido Galindo-Iranzo ${ }^{3}$, Sofia Kolida ${ }^{4}$, Lisa

Methven $^{5}$, Robert A. Rastall ${ }^{5}$, F. Javier Moreno*1, Oswaldo HernandezHernandez ${ }^{1}$

Institute of Food Science Research, CIAL (CSIC-UAM), Nicolás Cabrera 9, 28049 Madrid, Spain; ana.munoz@csic.es (A. M-L); javier.moreno@csic.es (F.J. M.); o.hernandez@csic.es (O. $\mathrm{H}-\mathrm{H})$

Consejo Nacional de Investigaciones Científicas y Técnicas (CONICET), Godoy Cruz 2290 CABA (C1425FQB), Argentina; silvanaazcarate@gmail.com (S.A) Institute of Physical Chemistry 'Rocasolano’ (IQFR-CSIC), Serrano 119, 28006 Madrid, Spain; rlebron@iqfr.csic.es (R. L-A); je.quintanilla@iqfr.csic.es (JE. Q-L); pgalindo@iqfr.csic.es (P. G-I) OptiBiotix Health plc, Innovation Centre, Innovation Way, Heslington, York, Y010 5DG, United Kingdom; skolida@optibiotix.com (S.K) Department of Food and Nutritional Sciences, The University of Reading, PO Box 226, Whiteknights, Reading, RG6 6AP, United Kingdom; l.methven@reading.ac.uk (L. M); r.a.rastall@reading.ac.uk (R. R)

* $\quad$ Correspondence: javier.moreno@csic.es; Tel.: +34-910017948 (F.J. M.) 
1 ABSTRACT: Luo-Han-Guo fruit extract (Siraitia grosvenorii), mainly composed of

2 mogroside V (50\%) could be considered a suitable alternative to free sugars, however, its

3 commercial applications are limited by its unpleasant off-notes. In the present work, a

4 central composite design method was employed to optimize the transglycosylation of a

5 mogroside extract by using cyclodextrin glucosyltransferases (CGTases) from three

6 different bacteriological sources (Paenibacillus macerans, Geobacillus sp and

7 Thermoanaerobacter $\mathrm{sp}$ ) considering various experimental parameters such as

8 maltodextrin and mogroside concentration, temperature, time of reaction, enzymatic

9 activity and pH. Product structures were determined by liquid chromatography coupled

10 to diode-array detector (LC-DAD), liquid chromatography-mass spectrometry (LC-ESI-

11 MS) and matrix-assisted laser desorption ionization time-of-flight mass spectrometry

12 (MALDI-TOF MS). Sensory analysis of glucosylated mogrosides showed an improvement

13 in flavor attributes relevant to licorice flavor and aftereffect. Consequently, an optimum

14 methodology was developed in order to produce new modified mogrosides more suitable

15 when formulating food products as free sugar substitutes.

17 KEYWORDS: sweetener, CGTase, glycosylation, sensorial profile, mogroside V. 


\section{INTRODUCTION}

According to the World Health Organization (WHO) non-communicable diseases (NCDs) caused an estimated 73\% of deaths in 2017 with obesity being one of the major risk factors for some NCDs such as cardiovascular disease and type 2 diabetes. ${ }^{1}$ One of the main factors leading to a high risk of obesity, and in consequence NCDs, is a high intake of free sugars.

An effective alternative to substitute the sweetness of free sugars in food is the use of high-intensity sweeteners (HIS). ${ }^{2}$ There are several HIS on the market that are generally recognized as safe (GRAS) including aspartame, acesulfame-K, neotame, sucralose, rebaudioside A, stevioside and, more recently, an extract of the Luo Han Guo fruit or monk fruit. $^{3}$

Monk fruit extract is formed by several compounds classified as curcubitane-type triterpenoid glycosides, which are formed by a mogrol aglycone glycosylated with glucose units. ${ }^{4}$ The main compound found is Mogroside V followed by Mogrosides IV and $\mathrm{VI}^{5}$ (Figure 1), which are known to exert a sweetness intensity 250-300 times higher than sucrose. ${ }^{3,6}$ On the contrary, mogrosides with fewer than three glycoside units are not sweet. ${ }^{7}$ Several works have reported the potential pharmacological properties and bioactivities of various naturally occurring mogrosides, such as antioxidant and antiinflammatory effects, ${ }^{8}$ antiproliferative activity, attenuation of liver fibrosis, ${ }^{9}$ and hypoglycaemic effects by inhibition of the intestinal maltase-glucoamylase, among others. ${ }^{10}$ However, most of these studies have been carried out using in vitro systems or animal trials, still, human interventions are needed to confirm all of these potentially

40 beneficial properties. Despite this, mogrosides could make a valuable substitute to free sugars with potential added bioactivities. ${ }^{11}$ 
Despite the use of mogrosides as HIS and the reported bioactive properties, there are few comprehensive studies on their flavor profiles, commonly identifying licorice aftertaste. ${ }^{12}$ Based on this, an improvement of flavor might be feasible by the glycosylation of mogrosides as previously reported by Yoshikawa et al. ${ }^{13}$. These authors used various cyclodextrin glycosyltransferases (CGTases, E.C 2.4.1.19) to glucosylate mogroside V, obtaining an addition of up to three units of glucose to the mogroside $\mathrm{V}$ moiety and reporting an improvement in the taste. CGTases are hexosyltransferases produced by different bacterial species and are widely used to catalyze the synthesis of cyclodextrins from starch and other related compounds. There are also other compounds used as acceptors in CGTase transglycosylation reactions with different purposes, such as polyphenols, ${ }^{14-16}$ vitamins, sugar alcohols ${ }^{17}$, and other natural sweeteners based on entkaurene derivative from Stevia rebaudiana. ${ }^{18}$

Considering that CGTases can recognize a number of acceptors with different structural features, including terpenoid compounds as steviol glycosides ${ }^{18,19}$, and that mogrosides are naturally occurring high-intensity sweeteners largely unexplored as substrates for enzymatic transglycosylation modification, this work addresses the potential improvement of the flavor profile of a mogroside extract (Mogroside V 50\% $\mathrm{w} / \mathrm{w}$ ) attained by enzymatic transglycosylation. Response surface methodology has been

60 used to assess the optimization of the experimental conditions. Unmodified and 61 glucosylated mogrosides were subjected to a comprehensive structural analysis by MALDI-TOF MS and LC-MS analyses together with a detailed flavor study by determining 16 flavor characteristics. 

MATERIALS AND METHODS

\section{Chemicals}

An extract from Luo-Han-Guo fruit (Siraitia grosvenorii) mainly formed by $50.33 \%$

moisture (as declared by the supplier) was purchased from MonkFruit Corp. (Libertyville,

USA). CGTases from Paenibacillus macerans, Geobacillus sp and Thermoanaerobacter sp

were used. Maltodextrin from maize starch (20 dextrose equivalent), acetonitrile (MS

grade), and formic acid (MS grade) were obtained from Sigma-Aldrich (St. Louis, USA).

Mogroside V standard was purchased from Biosynth Carbosynth (Reading, UK). White

granulated sugar (Tate and Lyle, London, UK) and water (Harrogate Spa mineral water)

for sensory analysis were purchased in local supermarkets in Reading (UK). All other reagents were obtained from Sigma-Aldrich (St Louis, USA) and ThermoFisher Scientific (San Jose, USA).

\section{Statistical experimental design for transglucosylation of mogrosides}

Optimization of transglucosylation reactions with the three CGTases was carried out by multivariate analysis (Software Design Expert 10.1, StatEase, USA), considering the effect of 6 different parameters that were concentration of maltodextrin (g/L), mogroside $\mathrm{V}(\mathrm{g} / \mathrm{L})$, enzymatic activity (U/g mogroside $\mathrm{V})$, temperature $\left({ }^{\circ} \mathrm{C}\right)$, reaction time $(\mathrm{h})$ and pH. First, the most influential parameters were established using a fractional factorial design $\left(2^{(6-2)}\right)$, and then a Central Composite Design (CCD) was used to obtain the optimum conditions for the glucosylation reactions. The assay precision was evaluated by 
86

87

88

89

90

91

92

93

94

95

the relative percentage error (RE) and the appropriate relative standard deviation (RSD) from the theoretical concentrations of mogroside $\mathrm{V}$ by external calibration.

89

\section{Liquid chromatography coupled to diode-array detector (LC-DAD) analysis}

Concentration of glucosylated mogrosides was determined by LC-DAD using a reversed-phase Poroshell $120 \mathrm{C} 18$ column $(250 \mathrm{~mm} \times 4.6 \mathrm{~mm}, 4 \mu \mathrm{m}$ particle size, $120 \AA$ pore size, Agilent Technologies, Palo Alto, USA) in an LC system (Agilent 1200 series, equipped with an autosampler, quaternary pump, column oven and DAD detector). The separation was performed at a flow rate of $0.7 \mathrm{~mL} / \mathrm{min}$ at $30^{\circ} \mathrm{C}$. The elution gradient using deionized water and acetonitrile changed from 90:10 (v:v) to 10:90 (v:v) over 50 min, then to initial conditions over 2 min and maintained for $10 \mathrm{~min}$ for conditioning.

(1)

Matrix-assisted laser desorption/ionization time-of-flight mass
spectrometry (MALDI-TOF MS) analysis

The molecular weight distribution of mogroside mixtures was determined by MALDI-TOF MS. Samples were diluted 1:100 in water (Milli-Q water, Millipore, Bedfor, USA), and $1 \mu \mathrm{L}$ of the diluted solutions were mixed with $3 \mu \mathrm{L}$ of the matrix consisting of 2,5-dihydroxybenzoic acid (>98\%, Fluka, Buchs, Switzerland) at a concentration of 10 $\mathrm{mg} / \mathrm{mL}$ in water. Then, $1 \mu \mathrm{L}$ of this mixture was spotted onto a flat stainless-steel sample plate and dried in air before analysis.

A Voyager DE-PRO mass spectrometer (Applied Biosystems, Foster City, USA), equipped with a nitrogen laser emitting at $337 \mathrm{~nm}$ and a delayed extraction ion source, was used for MALDI-TOF MS measurements. Ions generated by laser desorption of 
109

110

111

112

113

114

unmodified and glucosylated mogroside mixtures were introduced into the time of flight analyzer (1.3 m flight path) with an acceleration voltage of $25 \mathrm{kV}, 94 \%$ grid voltage, $0.075 \%$ ion guide wire voltage, and a delay time of 400 ns. Mass spectra were obtained over the $m / z$ range $500-4000$ in the linear positive ion mode as $[\mathrm{M}+\mathrm{Na}]^{+}$. External mass calibration was applied using the average $[\mathrm{M}+\mathrm{H}]^{+}$values of the components of the Calibration Mixtures 1 and 2 of Sequazyme Peptide Mass Standards Kits (Applied Biosystems).

\section{Liquid chromatography coupled to mass spectrometry (LC-MS) analysis}

LC-MS experiments were carried out on an Agilent 1100 Series LC system (Agilent Technologies, Palo Alto, USA) equipped with a quaternary pump, an autosampler, a column oven, and coupled to an HTC-Ultra ETD II ion trap mass spectrometer (Bruker Daltonics, Fremont, USA) using an ESI interface working in the negative-ion mode.

Unmodified and glucosylated mogroside mixtures were diluted 1:100 with water, and their separation $(5 \mu \mathrm{L})$ was performed at $35^{\circ} \mathrm{C}$ on a C18 HyPURITYTM column (100 mm x 2.1 mm, $3 \mu \mathrm{m}$ particle size, Thermo Fisher Scientific, San José, USA). A gradient consisting of 0.1\% (v:v) formic acid (analytical grade; Merck, Darmstadt, Germany) in water (eluent A) and acetonitrile (OPTIMA ${ }^{\circledR} \mathrm{LC} / \mathrm{MS}$ grade, ThermoFisher Scientific) containing $0.1 \%$ of formic acid (v:v) (eluent B) was applied at a flow rate of $0.2 \mathrm{~mL} / \mathrm{min}$ as follows: $10 \%$ B for $1 \mathrm{~min}, 10-50 \%$ B linear in $45 \mathrm{~min}, 50 \%$ B for $5 \mathrm{~min}, 50-90 \%$ B linear from 50 to $51 \mathrm{~min}, 90 \% \mathrm{~B}$ isocratic from 51 to $60 \mathrm{~min}$, ramped to original composition in $1 \mathrm{~min}$, then re-equilibration of the column for $10 \mathrm{~min}$.

The mass spectrometer spray voltage was set at $3.5 \mathrm{kV}$, the drying gas temperature at $350{ }^{\circ} \mathrm{C}$, and the skimmer voltage at $-40 \mathrm{~V}$. Nitrogen (99.5\% purity) was used as drying 
$133(10 \mathrm{~L} / \mathrm{min})$ and nebulizer (40 psi) gas. Full scan mass spectra were recorded between $\mathrm{m} / \mathrm{z}$ 134100 and 2000. Bruker Compass 1.2 software was used for LC-MS data acquisition and 135 processing.

\section{Mogroside fraction isolation for sensory analysis}

$\mathrm{cm}$ ) and washed with deionized water (1500 mL water). Both unmodified mogrosides and analysis. Removal of free carbohydrates was efficiently carried out and monitored by gas chromatography with flame-ionization detection (GC-FID).

\section{Sensory test procedure}

The sweetness intensity of the glucosylated mogrosides obtained under optimal synthesis conditions together with the unmodified mogrosides was evaluated using 10 experienced sensory evaluation panelists. The panelists were trained at the Sensory

Science Centre (Department of Food and Nutritional Sciences, University of Reading, UK).

150 The study was approved by the University of Reading Research Ethics Committee (UREC 151 study number $\left.16 \_19\right)$. Sensory analysis was performed in an air-conditioned (23-24 ํㅡ,

152 room temperature) sensory laboratory with individual booths and artificial daylight.

154 taste/flavor, bitter taste, licorice flavor, sour taste, cooked sugar flavor, cooling sensation, 155 cardboard/stale, metallic, salty taste, crusty bread flavor, perfume flavor, sweet aftertaste, 
156 bitter aftertaste, licorice aftereffect, and cooling aftertaste), followed by training focused

157

158

159

160

161

162

163

164

165

166

167

168 dose-response curve, the linear regression for which was Perceived Sweetness $=(1.55 \times$

178 Sucrose Concentration $(\mathrm{g} / \mathrm{L}))-22.5\left(\mathrm{r}^{2}=0.99\right)$. The mean sweetness ratings for each on ensuring each panelist could reliably score sweetness relative to four sucrose standards $(20,30,60$ and $80 \mathrm{~g} / \mathrm{L})$. The average panel ratings for these standards were 10, 35,75 , and 100 respectively on a $0-100$ line scale, and so these four positions were used as anchors to provide a structured scale on which to rate the sweetness of all samples. All other attributes were scored as relative values using unstructured line scales (0-100). Due to the limited sample availability, each panelist was presented with only $0.5 \mathrm{~mL}$ of sample for each scoring session. Therefore, training additionally focused on ensuring panelists were able to sip this small sample volume from a $30 \mathrm{~mL}$ transparent polystyrene cup and allow it to flow over the top of their tongue before swallowing and scoring sweetness accurately. Palate cleansing before and between sample scoring was done using filtered water and low salt crackers (Carr's water crackers, United Biscuits Ltd., Hayes, UK).

Samples were prepared in mineral water (Harrogate Spa mineral water) and labeled with random 3-digit codes. Sample order presentation was done in a balanced monadic sequential manner. An initial screening was carried out to choose the most appropriate mogroside concentration, this was concluded as $0.24 \mathrm{~g} / \mathrm{L}$.

The sucrose standards were presented at the start of each panel rating session for re-familiarisation in order to enable the panelists to score the sweetness of the samples accurately against the standard anchors.

The mean sweetness ratings of the four sucrose standards were used to plot a sample were converted to equivalent sweetness (ES) values using this equation. 

panelists were treated as random effects and samples as fixed effects. The main effects were tested against the sample by assessor interaction. Multiple pairwise comparisons

183 were carried out using Fisher's LSD and a significant difference was declared at an alpha 184 risk of 5\% ( $\mathrm{p} \leq 0.05)$. Data analysis was carried out using Senpaq software (Qi Statistics, 185 Reading, UK).

\section{RESULTS}

\section{Optimization of mogroside glucosylation parameters}

A screening fractional factorial design $2^{(6-2)}$ was first used to study the synthesis of 190 glucosylated mogrosides with the three different CGTases employed. This screening 191 allowed us to evaluate the effect of 6 experimental factors (concentration of maltodextrin, 192 concentration of unmodified mogrosides, enzymatic activity, temperature, time, and pH), 193 using the concentration of glucosylated mogrosides $(\mathrm{mg} / \mathrm{mL})$ as a response variable. 194 Table 1 shows the runs carried out and the responses obtained for each enzyme, 195 calculated from LC-DAD analyses. The average RE of mogroside $\mathrm{V}$ was $2.3 \%$ (RSD = $1.8 \%$, $196 \mathrm{n}=4$ ) suggesting an acceptable accuracy and precision for the quantitation of the samples. The highest concentration $(14.0 \mathrm{mg} / \mathrm{mL})$ of glucosylated mogrosides was obtained 198 using the CGTase from Thermoanaerobacter sp (Table 1), which was chosen to continue 199 with synthesis optimization. 
Figure 2 shows the Pareto chart illustrating the analysis of variance (ANOVA) and 201 p-value, which were used to evaluate the significance of the effect. Positive values (green 202 bars) denote a directly proportional relationship of the variable with the response; 203 whereas negative values (red bars) reflect an inverse relationship. The horizontal line 204 corresponds to the t-value at a significance level of 5\%. The concentration of unmodified 205 mogrosides and the enzyme activity had a significantly positive effect on the response 206 (glucosylated mogrosides concentration). Consequently, these two factors (concentration 207 of unmodified mogrosides and the enzyme activity) were chosen to carry out the Central 208 Composite Design (CCD) optimization.

Maltodextrin concentration, temperature, and time were fixed at their lowest 210 value, $5 \mathrm{~g} / \mathrm{L}, 50^{\circ} \mathrm{C}$, and $1 \mathrm{~h}$ respectively, and the $\mathrm{pH}$ value was adjusted to 5 . The CCD was 211 composed of 11 experimental points with 3 replicates in the central point. The experimental data provided by CCD design were analyzed by regression analysis. The 213 relationship between the response evaluated and the variables were fitted into the 214 polynomial Equation 1. unmodified mogrosides $)+0.43 *$ unmodified mogrosides $(\mathrm{mg} / \mathrm{mL})$ Equation 1 Analysis of variance (ANOVA) was carried out to justify the significance and 218 adequacy of the regression model fit. Statistical significance of the model was established 219 at $\mathrm{p} \leq 0.05$. The obtained model F-values $<0.03$ for the response indicate that the mode 220 was highly adequate and significant. Likewise, the determination coefficient (R-squared) 221 of the model was 0.98 for synthesized glucosylated mogrosides. The coefficient of 222 variation (CV \%) had lower values than 10\% showing that the variation was acceptable 223 and satisfactory. 
The optimum concentration of unmodified mogrosides and enzymatic activity were determined to maximize the yield of glucosylated mogrosides (mg/mL). The response surface obtained for the global desirability function (D) is presented in Figure S1. The coordinates producing the maximum desirability value $(D=1)$ were $56.7 \mathrm{~g} / \mathrm{L}$ for the concentration of unmodified mogrosides and $6.5 \mathrm{U} / \mathrm{g}$ of mogroside $\mathrm{V}$ for the enzymatic activity.

The individual response values and their respective confidence intervals are depicted in Table 2. In order to validate this predictive model, optimal conditions were experimentally assessed through three replicates which showed no significant differences with the theoretical results. Finally, these conditions were selected to produce higher quantities of synthesized glucosylated mogrosides to be structurally characterized and for the sensory analysis.

\section{Structural characterization by mass spectrometry}

According to Table 1, the CGTase from Thermoanaerobacter sp. generated the highest concentration of synthesized glucosylated mogrosides determined by LC-DAD. In order to qualitatively evaluate the polymerization activity of the three CGTases used in this study, MALDI-TOF MS analyses were carried out and compared with that of the unmodified mogrosides (Figure 3). The three CGtases showed a high glucosylation 243 activity of up to 12 units of glucose. Deglucosylation activity was not detected and 244 significant amounts of mogrosides IV or III $\left([\mathrm{M}+\mathrm{Na}]^{+} 1148.3,986.2\right.$, respectively) were not produced.

The initial mixture of unmodified mogrosides was properly characterized. using 247 LC-DAD and LC-MS, and was composed of 50.2\% mogroside V, 31.9\% mogroside VI (and 
248 other unknown minor mogrosides), 10.2\% mogroside IV, and 7.7\% mogroside III, which 249 were identified by the retention times and their corresponding molecular ions (Figure 250 S2). Due to the lack of structural information generated by LC-DAD and the impossibility 251 of identifying if mogroside $\mathrm{V}$ and/or the other minor mogrosides present in the mixture 252 are glucosylated, an LC-MS analysis was carried out on the enzymatically modified sample 253 (Figure 4). Since the MALDI-TOF MS analysis showed that the three CGTases produce a 254 similar glucosylation pattern (up to 12 glucose units), the LC-MS analysis was carried out 255 on the sample obtained using optimized conditions (CGTase from Thermoanaerobacter $256 \mathrm{sp})$.

The results shown in Figure 4 confirmed the glucosylation of the mogroside 258 structures by the identification of new peaks with lower retention times (Figure 4B) when 259 compared with the initial mogroside mixture (Figure $4 \mathrm{~A}$ ), and by the $[\mathrm{M}-\mathrm{H}]^{-}$ions corresponding to the newly synthesized mogrosides with up to 4 new glucose units. Due

261 to the low sensitivity of LC-MS to higher molecular weight compounds, mogrosides with 262 more than 9 glycosides units were not identified by this technique. However, the LC-MS 263 profiles revealed seven potential mogroside IV isomers $(25.8,26.3,26.7,27.0,27.2,27.6$, 264 and $27.9 \mathrm{~min})$ in the unmodified mogroside mixture $(\mathrm{m} / z$ 1123.6; Figure 5$)$. To the best 265 of our knowledge, only two mogroside IV structures have been described, mogroside IVe 266 and mogroside IVa. This is the first time that five additional different potential mogrosides 267 IV have been described. LC-MS data allowed us to determine the ratio of mogroside $\mathrm{V}$ and the mogroside IV 269 in the unmodified and glucosylated mogroside samples. The most abundant mogroside 270 was mogroside $\mathrm{V}(\mathrm{m} / \mathrm{z}$ 1285.5) followed by the mogroside IV isomers $(\mathrm{m} / \mathrm{z} 1123.0$, Figure 271 5). The area ratio of mogroside IV / mogroside V was similar in two of the mogroside IV 
isomers, indicating that the rate of glucosylation is similar between mogroside $\mathrm{V}$ and these mogroside IV isomers. However, four of the mogroside IV isomers were more readily

274 glucosylated by the CGTase than mogroside V. Also, after the enzymatic reaction, a new 275 mogroside IV (Figure 4; $30.02 \mathrm{~min}$ ) was synthesized. This new mogroside IV could be a 276 glucosylated form of the minor mogroside III isomers presented in the initial mixture. 277 Interestingly, Figure 5 also shows similar behaviour in mogroside III compared with 278 mogroside IV. The main two mogroside III isomers decreased and one of mogroside III 279 isomers increased (Figure 5; $29.70 \mathrm{~min}$ ).

\section{Sensory profiling}

Mogroside V is reported to have a sweetness potency approximately 250 times that of sucrose. ${ }^{12}$ Previous literature ${ }^{20}$ has shown that bitterness and other off-flavors exist in mogroside extract ( $50 \%$ mogroside $\mathrm{V}$ ). In this study, we decided to test solutions at equivalent sweetness levels above $2 \%$ sucrose to ensure that we were able to evaluate both sweetness and bitterness in levels suitable for potential food application. The glucosylated mogroside samples were purified using HP-20 resin, resulting in a purity higher than $98 \% \mathrm{w} / \mathrm{w}$. This purification technique allowed to eliminate the free

289 carbohydrates, i.e. glucose, maltose and maltodextrins, present after the synthesis (as monitored by GC-FID) and the resulting product was only based on mogrosides

291 (unmodified and enzymatically glucosylated forms).

For the initial tasting, sucrose standards were prepared at 4, 6, 8, and $10 \%(\mathrm{w}: \mathrm{v})$.

293 Aiming for 8\% sucrose, equivalent unmodified mogrosides and the glucosylated sample 294 were prepared at $0.32 \mathrm{~g} / \mathrm{L}$. From the initial tasting we concluded that we needed to reduce 295 the sucrose standard range to $2,4,6$, and $8 \%$ as the $10 \%$ sample was too extreme. The 
296 unmodified mogroside sample and the respective enzymatically modified sample were 297 considered too sweet relative to the sucrose standards, therefore they were reduced in 298 concentration to $0.24 \mathrm{~g} / \mathrm{L}$.

Of the 16 attributes rated, 5 of them differed significantly from sample to sample 300 (Figure 6). In general, licorice and metallic flavors were significantly decreased by the 301 enzymatic modification, although so was the sweet taste. All other specifically defined 302 taste and flavor attributes were rated at low levels. The lowering power of glucosylation 303 on sweetness has been observed previously. ${ }^{13}$ This decrease is in inverse proportion to 304 the amount of glucose monomer attached to the aglycone. However, in the case of the 305 mogrosides obtained after the glucosylation reaction, the sweetness potency is only 1.2 306 times lower than in the unmodified mixture (Table 3). Consequently, the glucosylated 307 mogroside may still be considered a high-intensity sweetener.

\section{DISCUSSION}

It has been noted that glycosylation of natural sweeteners such as steviol 311 glycosides ${ }^{18,19}$ and mogrosides ${ }^{13}$ can improve their flavor profile. In the case of 312 mogrosides, very few reports have analyzed the off-flavors of these compounds. Tan et 313 al. $^{20}$ reported a high level of bitterness and metallic flavor in mogroside extract 314 (mogroside $\mathrm{V}, 50 \%$ ), similar to that found in rebaudioside A, one of the major steviol 315 glycosides present in commercial mixtures. On the contrary, Yoshikawa et al. ${ }^{13}$ and Kim 316 et al. ${ }^{21}$ reported a relatively low bitter taste, but a high intensity of licorice flavor and 317 general aftertaste. These discrepancies could be due to the different proportion of natural 318 mogrosides and their concentrations, or as a result of the sensory analysis performed. 
319 However, it is evident there is an undesirable aftertaste in the tested mogroside mixtures. 320 Also, it is important to consider that the work of Yoshikawa et al. obtained 321 transglycosylated products with only up to 1 to 3 additional glucose residues on the 322 mogroside V structure. ${ }^{13}$ The improved taste profile of triterpenoid saponins derived from mogrosides 324 using a biosynthetic pathway based on enzymes from microbial sources would provide a 325 promising and green approach for supplying new consumer-friendly products to the 326 market. $^{22}$ Several attempts have used enzymes such as $\beta$-glucosidases, pectinases, 327 cellulases, and uridine diphosphate glycosyltransferases (UGTs) in order to produce 328 specific mogrosides. ${ }^{11,22,23}$ Cyclodextrin glycosyltransferases (EC 2.4.1.19) are an 329 excellent approach to the modification of mogrosides due to their use of readily available 330 glycosyl donors (starch or maltodextrin), food-grade status, and commercial availability. 331 CGTases are mainly used in the food industry to catalyze the conversion of starch in 332 cyclodextrins. These enzymes can also act as glucosyltransferases with different 333 acceptors, such as steviol glycosides. ${ }^{19}$

Due to the nature of the catalytic activity of the CGTases, glucose units are 335 transferred to an acceptor, mainly low molecular weight carbohydrates via $\alpha-(1-4)$ 336 linkages. The mogroside V (CAS 88901-36-4) has five glucose units attached to the 337 aglycone (mogrol). The elongation could hypothetically occur either on the glucose chain 338 attached to the $\mathrm{C}-3$ of the mogrol structure, or on the glucose chain attached to the C-24 339 chain of the mogrol structure (Figure 1), based on the additional glucose of other natural 340 mogrosides such as mogrosides VI, VIa1, and VIa, respect to the mogrosides V. ${ }^{24}$ 341 Moreover, Wang et al. ${ }^{25}$ carried out a transglycosylation method for which they formed 342 new mogroside compounds and other known natural mogrosides. Based on these findings 
343 and on the mechanisms of transglycosylation activity of bacterial CGTases, the 344 transglycosylation of the present samples could take place through the transfer of glucose 345 residues in an $\alpha$-orientation to the accessible (terminal) glucoses attached in either the C3463 or the C-24 of the mogrol core. Whereas the preferable regioselectivity of bacterial 347 CGTases could point out through the formation of $\alpha-(1-4)$-linkages, other possible 348 glycosidic bonds, such as $\alpha-(1-6)$ and $\alpha-(1-2)-l i n k a g e s$ should not be fully dismissed ${ }^{25}$. 349 According to Figure 4B, three peaks corresponded to mogroside VI. These could be 350 consequences of the glucosylation of two different C-4 on the glucoses presented in the 351 mogroside $\mathrm{V}$, however, the high abundance of one mogroside VI isomer over the other one 352 is indicative that one C-4 is more prone to being glucosylated.

In this study, for the first time, was reported a glucosylation of the mogrosides in a commercial sweetener mixture (Mogroside V $50 \% \mathrm{w} / \mathrm{w}$ ) by up to 12 glucose units, resulting in an aglycone (mogrol) glucosylated by up to 17 glucose units. Interestingly, it was found that the isomers of mogrosides present in the initial mixture are glucosylated 357 in different ratios. Glucosylation significantly improved the sensory profile. However, to ensure that this new product could suppose an optimal alternative to sugar, future research dealing with metabolic studies following oral ingestion, including the potential 360 impact on gut microbiota composition and activity, should be considered. Furthermore, the use of response surface methodology approach allowed for optimization of conditions for the cost-effectiveness of the new modified mogroside mixture production, which could be easily scaled-up to pilot or industrial level. ${ }^{23}$ on experimental design methodologies, which is reported to produce highly glucosylated mogroside $\mathrm{V}$, using a commercial mixture of mogrosides (50\% mogroside $\mathrm{V}$ ), 
maltodextrin and CGTases that are widely used in the food industry as starting materials.

368

369

370

371

372

373

374

376

This modification allows the production of a natural sweetener with improved flavor, formed from a mixture of mogrol with up to 17 glucose units. This optimized mogroside mixture has an improved flavor with lower licorice and metallic flavors, which are characteristic of the unmodified mogroside mixtures currently found on the market.

Funding: This research was funded by Optibiotix Health plc (York, UK), by the Spanish Ministry of Science, Innovation and Universities (project AGL2017-84614-C2-1-R) and by FEDER-Spanish Ministry of Science and Innovation (project RTI2018-101273-J-I00).

Conflicts of Interest: The authors declare the following competing financial interest(s): S.K is the R\&D Director of Optibiotix Health plc.

\section{SUPPORTING INFORMATION}

This material is available free of charge via the Internet at http://pubs.acs.org:Threedimensional plot showing the effect of initial unmodified mogrosides and the enzymatic activity on the desirability to maximize the concentration of synthesized glucosylated mogrosides (Figure S1); LC-DAD chromatogram of unmodified Luo-Han-Guo fruit extract. The identified compounds correspond to Mogrosides V, VI*, IV, and III. *Other minor nonidentified mogrosides (Figure S2A); LC-MS spectrum corresponding to the cucurbitane triterpene glycosides found in the unmodified Luo-Han-Guo fruit extract: $1285.6 \mathrm{~m} / \mathrm{z}-$ Mogroside V, 1447.6 m/z - Mogroside VI, 1123.6 m/z - Mogroside IV, and 965.4 m/z Mogroside III (Figure S2B). 


\section{REFERENCES}

390

(1) World Health Organization Guideline: Sugars intake for adults and children;

391 Organization, W.H., Ed.; World Health Organization, 2015; ISBN 9789241549028.

392 (2) Di Monaco, R.; Miele, N.A.; Cabisidan, E.K.; Cavella, S. Strategies to reduce sugars in 393 food. Curr. Opin. Food Sci. 2018, 19, 92-97.

394 (3) Mooradian, A.D.; Smith, M.; Tokuda, M. The role of artificial and natural sweeteners 395 in reducing the consumption of table sugar: A narrative review. Clin. Nutr. 2017, 18, 1-8.

396 (4) Chiu, C.H.; Wang, R.; Lee, C.C.; Lo, Y.C.; Lu, T.J. Biotransformation of mogrosides 397 from Siraitia grosvenorii swingle by Saccharomyces cerevisiae. J. Agric. Food Chem. 2013, $398 \quad 61,7127-7134$.

399 (5) Gong, X.; Chen, N.; Ren, K.; Jia, J.; Wei, K.; Zhang, L.; Lv, Y.; Wang, J.; Li, M. The fruits 400 of Siraitia grosvenorii: a review of a chinese food-medicine. Front. Pharmacol. 2019, 10, 4011400.

402 (6) Pawar, R.S.; Krynitsky, A.J.; Rader, J.I. Sweeteners from plants-with emphasis on 403 Stevia rebaudiana (Bertoni) and Siraitia grosvenorii (Swingle). Anal. Bioanal. Chem. 2013, $404 \quad 405,4397-4407$.

405 (7) Matsumoto, K.; Kasai, R.; Ohtani, K.; Tanaka, O. Minor cucurbitane-glycosides from 406 fruits of Siraitia grosvenori (Cucurbitaceae). Chem. Pharm. Bull. (Tokyo). 1990, 38, 2030$407 \quad 2032$.

408 (8) Liu, C.; Dai, L.; Liu, Y.; Dou, D.; Sun, Y.; Ma, L. Pharmacological activities of 409 mogrosides. Future Med. Chem. 2018, 10, 845-850. 
410 (9) Cao, F.; Zhang, Y.; Li, W.; Shimizu, K.; Xie, H. International Immunopharmacology

411 Mogroside IVE attenuates experimental liver fibrosis in mice and inhibits HSC activation

412 through downregulating TLR4-mediated pathways. Int. Immunopharmacol. 2018, 55, 413 183-192.

414 (10) Suzuki, Y. a; Murata, Y.; Inui, H.; Sugiura, M.; Nakano, Y. Triterpene glycosides of 415 Siraitia grosvenori inhibit rat intestinal maltase and suppress the rise in blood glucose 416 level after a single oral administration of maltose in rats. J. Agric. Food Chem. 2005, 53, $417 \quad 2941-6$.

418 (11) Murata, Y.; Ogawa, T.; Suzuki, Y. a.; Yoshikawa, S.; Inui, H.; Sugiura, M.; Nakano, Y. 419 Digestion and absorption of Siraitia grosvenori triterpenoids in the rat. Biosci. Biotechnol. Biochem. 2010, 74, 673-676.

421 (12) Lindley, M.G. Natural high-potency sweeteners. In Sweeteners and Sugar 422 Alternatives in Food Technology; Wiley Online Books; Wiley-Blackwell: Oxford, UK, 2012; pp. 185-212 ISBN 9781118373941.

424 (13) Yoshikawa, S.; Murata, Y.; Sugiura, M.; Kiso, T.; Shizuma, M.; Kitahata, S.; Nakano, H. 425 Transglycosylation of mogroside $\mathrm{V}$, a triterpene glycoside in siraitia grosvenori, by cyclodextrin glucanotransferase and improvement of the qualities of sweetness. J. Appl. 427 Glycosci. 2005, 52, 247-252.

428 (14) Choung, W.J.; Hwang, S.H.; Ko, D.S.; Kim, S.B.; Kim, S.H.; Jeon, S.H.; Choi, H.D.; Lim, 429 S.S.; Shim, J.H. Enzymatic synthesis of a novel kaempferol-3-o- $\beta$-d-glucopyranosyl-(1 $\rightarrow 4)-$ $430 \quad$ o- $\alpha$-d-glucopyranoside using cyclodextrin glucanotransferase and its inhibitory effects on 431 aldose reductase, inflammation, and oxidative stress. J. Agric. Food Chem. 2017, 65, 27604322767. 
433 (15) Marié, T.; Willig, G.; Teixeira, A.R.S.; Gazaneo Barboza, E.; Kotland, A.; Gratia, A.; 434 Courot, E.; Hubert, J.; Renault, J.H.; Allais, F. Enzymatic synthesis of resveratrol $\alpha$ 435 glycosides from $\beta$-cyclodextrin-resveratrol complex in water. ACS Sustain. Chem. Eng. $4362018,6,5370-5380$.

437 (16) Gonzalez-Alfonso, J.L.; Leemans, L.; Poveda, A.; Jiménez-Barbero, J.; Olmo 438 Ballesteros, A.; Plou, F.J. Efficient $\alpha$-glucosylation of epigallocatechin gallate catalyzed by 439 cyclodextrin glucanotransferase from Thermoanaerobacter sp. J. Agric. Food Chem. 2018, $440 \quad 66,7402-7408$.

441 (17) Qi, Q.; Zimmermann, W. Cyclodextrin glucanotransferase: from gene to 442 applications. Appl. Microbiol. Biotechnol. 2005, 66, 475-485.

443 (18) Muñoz-Labrador, A.; Azcarate, S.; Lebrón-Aguilar, R.; Quintanilla-López, J.E.; 444 Galindo-Iranzo, P.; Kolida, S.; Methven, L.; Rastall, R.A.; Moreno, F.J.; Hernandez445 Hernandez, 0. Transglycosylation of Steviol Glycosides and Rebaudioside A: Synthesis 446 Optimization, Structural Analysis and Sensory Profiles. Foods 2020, 9(12), E1753.

447 (19) Gerwig, G.J.; te Poele, E.M.; Dijkhuizen, L.; Kamerling, J.P. Stevia Glycosides: 448 Chemical and Enzymatic Modifications of Their Carbohydrate Moieties to Improve the 449 Sweet-Tasting Quality. Adv. Carbohydr. Chem. Biochem. 2016, 73, 1-72.

450 (20) Tan, V.W.K.; Wee, M.S.M.; Tomic, O.; Forde, C.G. Temporal sweetness and side tastes 451 profiles of 16 sweeteners using temporal check-all-that-apply (TCATA). Food Res. Int. $452 \quad 2019,121,39-47$.

453 (21) Kim, M.J.; Yoo, S.H.; Jung, S.; Park, M.K.; Hong, J.H. Relative sweetness, sweetness 454 quality, and temporal profile of xylooligosaccharides and luo han guo (Siraitia 455 grosvenorii) extract. Food Sci. Biotechnol. 2015, 24, 965-973. 
456 (22) Zhao YJ, Li C. Biosynthesis of plant triterpenoid saponins in microbial cell factories. 457 J Agric Food Chem. 2018, 66, 12155-12165.

458 (23) Virly, Chiu CH, Tsai TY, Yeh YC, Wang R. Encapsulation of $\beta$-glucosidase within PVA 459 fibers by CCD-RSM-guided coelectrospinning: a novel approach for specific mogroside 460 sweetener production. J. Agric. Food Chem. 2020, 68, 11790-11801.

461 (24) Charan, R.; Devkota, K.P.; Bhanti, M.; Vadla, N. C.; Ma, G.; Walsh, A.; Harrigan, G.; 462 Prakash, I. Mogroside Via1, new isomer of mogroside VI isolated from Luo Han Guo. IOSR 463 J. Appl. Chem. 2019, 12, 1-7.

464 (25) Wang, L.; Yang, Z.; Lu, F.; Liu, J.; Song, Y.; Li, D. Cucurbitane glycosides derived from 465 mogroside IIE: structure-taste relationships, antioxidant activity, and acute toxicity. 466 Molecules 2014, 19, 12676-12689.

467 (26) Tardioli, P. W.; Zanin, G. M.; de Moraes, F. F. Characterization of 468 Thermoanaerobacter cyclomaltodextrin glucanotransferase immobilized on glyoxyl469 agarose. Enzyme Microb. Technol. 2006, 39, 1270- 1278. 

FIGURE CAPTIONS

472 Figure 1. Structures of triterpenoid compounds isolated from S. grosvenorii.

473 Figure 2. Pareto chart showing individual and interaction effects of the response evaluated 474 (synthesized glucosylated mogrosides using CGTase from Thermoanaerobacter sp. (A) 475 maltodextrin concentration, (B) initial mogrosides concentration, (C) enzymatic activity, (D) 476 temperature, (E) time and (F) pH. Using a confidence value of $\mathrm{p}=0.05$, based on a null hypothesis 477 test, values exceeding this limit (horizontal line) are considered as significant to the response 478 values.

479 Figure 3. MALDI-TOF MS profiles of unmodified mogrosides (a) and after the glucosylation under 480 optimized conditions, using CGTases from Thermoanaerobacter sp (b), Paenibacillus macerans (c) 481 and Geobacillus sp (d). Mog: Mogrol; Glc:glucose.

482 Figure 4. LC-MS ion traces profiles of the unmodified mogrosides mixture (A) and the 483 glucosylated mixture obtained under optimized conditions using CGTases from 484 Thermoanaerobacter sp (B).

485 Figure 5. LC-MS ion traces profiles of the initial unmodified mogrosides mixture (-, black line) 486 and the glucosylated mixture (-, red line) obtained under optimized conditions using CGTases 487 from Thermoanaerobacter sp.Figure 6. Mean panel ratings of attributes that either differed 488 significantly between samples or where mean rating $>10$. Attributes that were not significantly 489 different between samples or rated $<10$ were Bitter taste, Sour taste, Salty taste, Cooling 490 sensation, Stale flavor, Crusty Bread flavor, Perfume, Bitter aftertaste, and Cooling aftereffect.

491 Figure 6. Mean panel ratings of attributes that either differed significantly between 492 samples or where mean rating $>10$. Attributes that were not significantly different 493 between samples or rated $<10$ were Bitter taste, Sour taste, Salty taste, Cooling sensation, 494 Stale flavor, Crusty Bread flavor, Perfume, Bitter aftertaste, and Cooling aftereffect. 


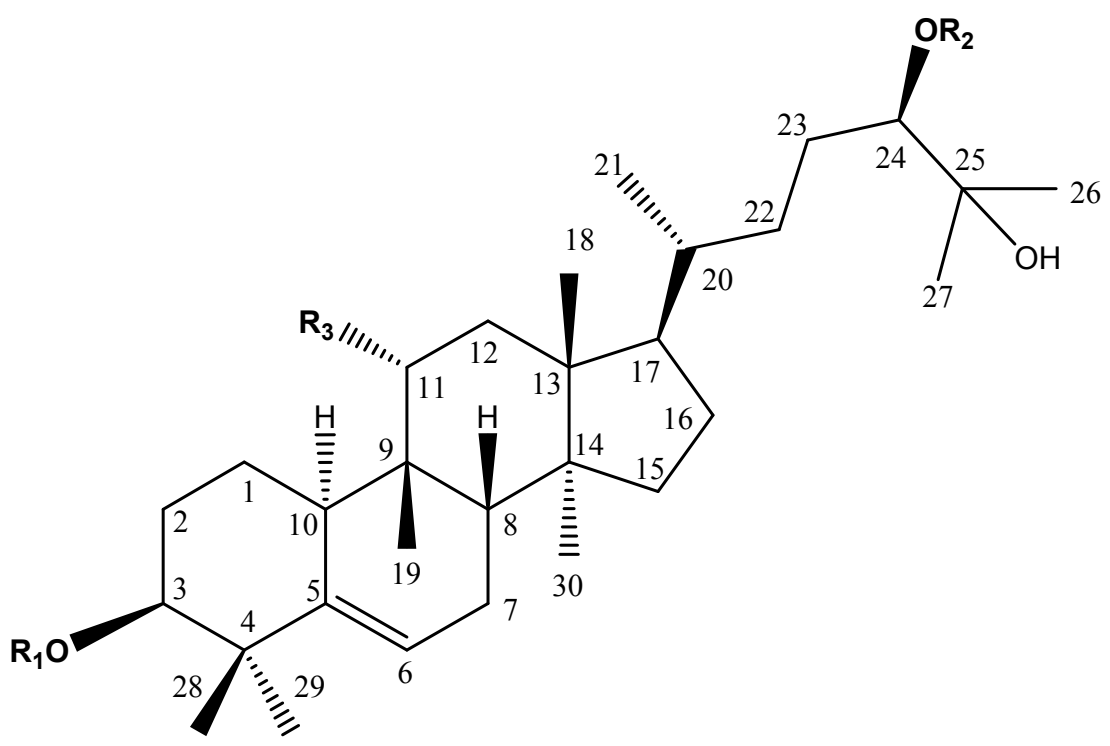

\begin{tabular}{cccc} 
Compound name & $\mathbf{R}_{\mathbf{1}}$ & $\mathbf{R}_{\mathbf{2}}$ & $\mathbf{R}_{\mathbf{3}}$ \\
\hline Mogrol & $\mathrm{H}$ & $\mathrm{H}$ & $\alpha-\mathrm{OH}$ \\
\hline Mogroside IA & $\mathrm{H}$ & $\mathrm{Glc}(\beta 1$ & $\mathrm{H}_{2}$ \\
\hline Mogroside IE1 & $\mathrm{Glc}(\beta 1$ & $\mathrm{Glc}(\beta 1-6) \mathrm{Glc}(\beta 1$ & $\alpha-\mathrm{OH}$ \\
\hline Mogroside IIA1 & $\mathrm{H}$ & $\mathrm{H}$ & $\alpha-\mathrm{OH}$ \\
\hline Mogroside IIA2 & $\mathrm{Glc}(\beta 1-6) \mathrm{Glc}(\beta 1$ & $\mathrm{Glc}(\beta 1$ & $\alpha-\mathrm{OH}$ \\
\hline Mogroside IIE & $\mathrm{Glc}(\beta 1$ & $\mathrm{Glc}(\beta 1-6)[\mathrm{Glc}(\beta 1-2)] \mathrm{Glc}(\beta 1$ & $\alpha-\mathrm{OH}$ \\
\hline Mogroside IIIAl & $\mathrm{H}$ & $\mathrm{Glc}(\beta 1$ & $\alpha-\mathrm{OH}$ \\
\hline Mogroside IIIA2 & $\mathrm{Glc}(\beta 1-6) \mathrm{Glc}(\beta 1$ & $\mathrm{Glc}(\beta 1-2) \mathrm{Glc}(\beta 1$ & $\alpha-\mathrm{OH}$ \\
\hline Mogroside IIIE & $\mathrm{Glc}(\beta 1$ & $\mathrm{Glc}(\beta 1-6) \mathrm{Glc}(\beta 1$ & $\alpha-\mathrm{OH}$ \\
\hline Mogroside IVA & $\mathrm{Glc}(\beta 1-6) \mathrm{Glc}(\beta 1$ & $\mathrm{Glc}(\beta 1$ & $\alpha-\mathrm{OH}$ \\
\hline Mogroside IVE & $\mathrm{Glc}(\beta 1-6) \mathrm{Glc}(\beta 1$ & $\mathrm{Glc}(\beta 1-6)[\mathrm{Glc}(\beta 1-2)] \mathrm{Glc}(\beta 1$ & $\alpha-\mathrm{OH}$ \\
\hline Mogroside V & $\mathrm{Glc}(\beta 1-6) \mathrm{Glc}(\beta 1$ & $\mathrm{Glc}(\beta 1-6)[\mathrm{Glc}(\beta 1-2)] \mathrm{Glc}(\beta 1$ & $\alpha-\mathrm{OH}$ \\
\hline Mogroside VI & $\mathrm{Glc}(\beta 1-6)[\mathrm{Glc}(\beta 1-2)] \mathrm{Glc}(\beta 1$ & &
\end{tabular}

Figure 1. 
Synthesized glucos ylated mogrosides $(\mathrm{mg} / \mathrm{mL})$

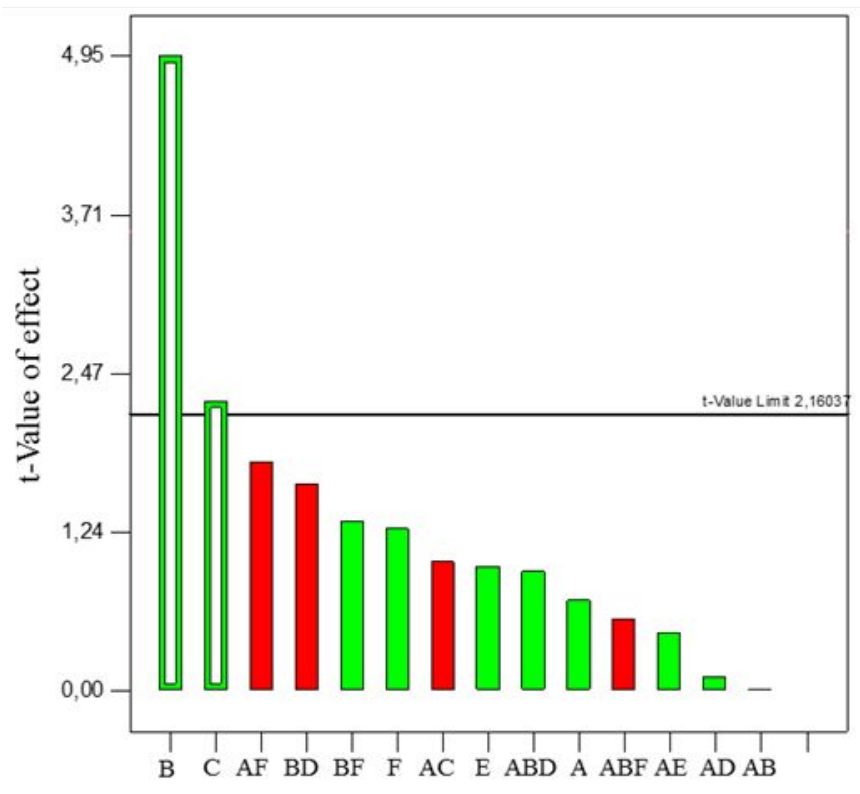

Figure 2. 


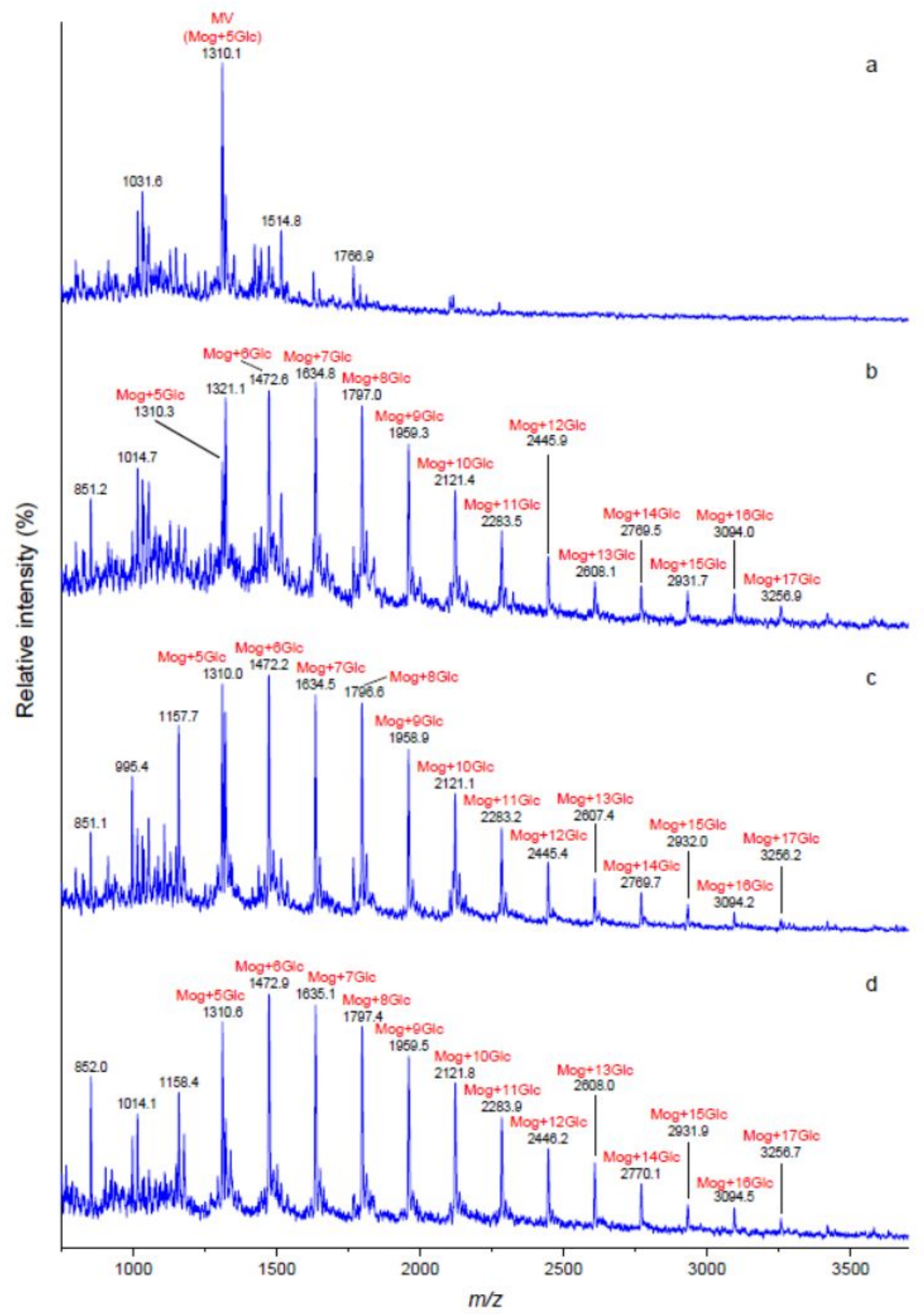

Figure 3. 
A

B

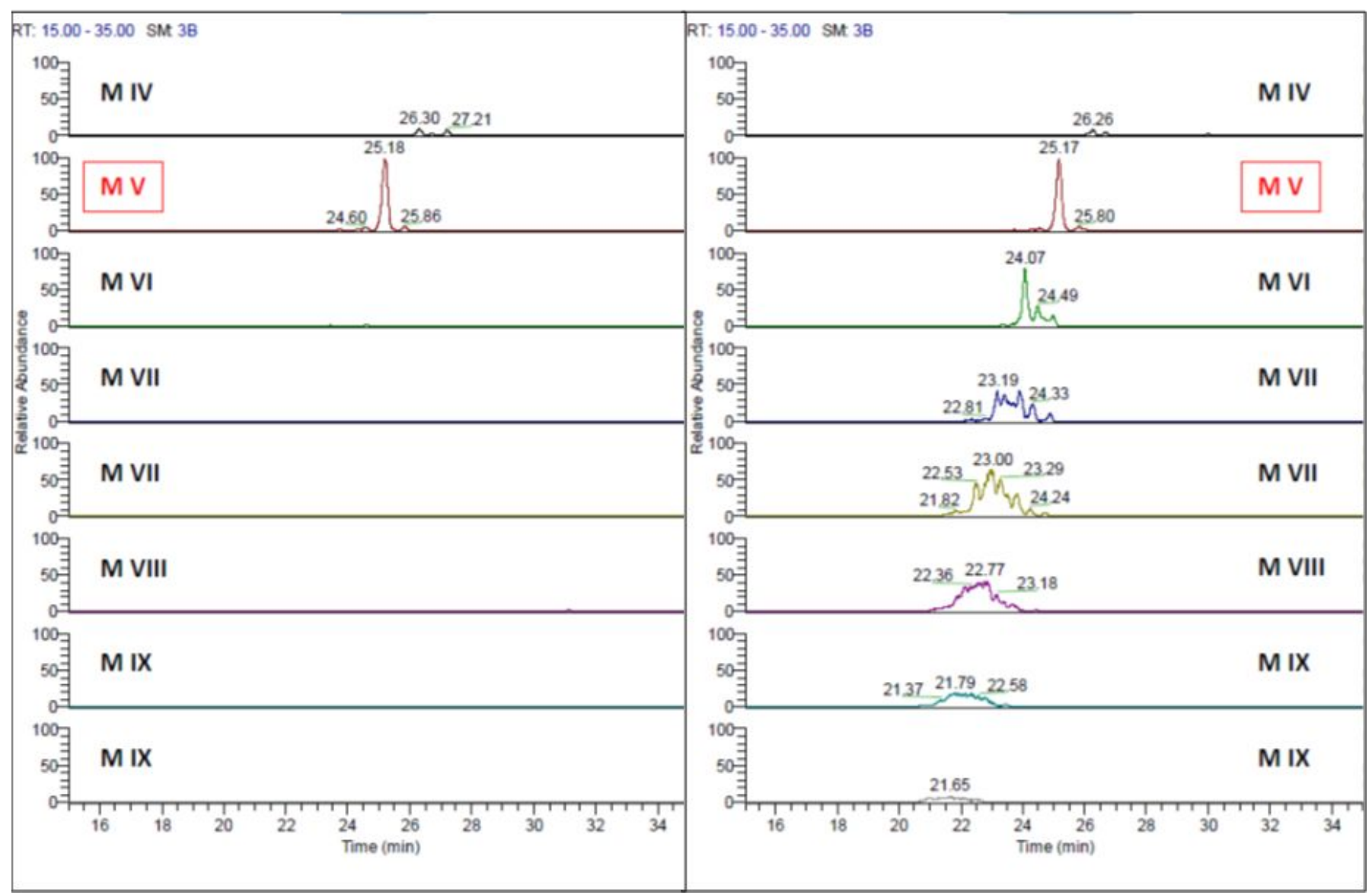

Figure 4. 


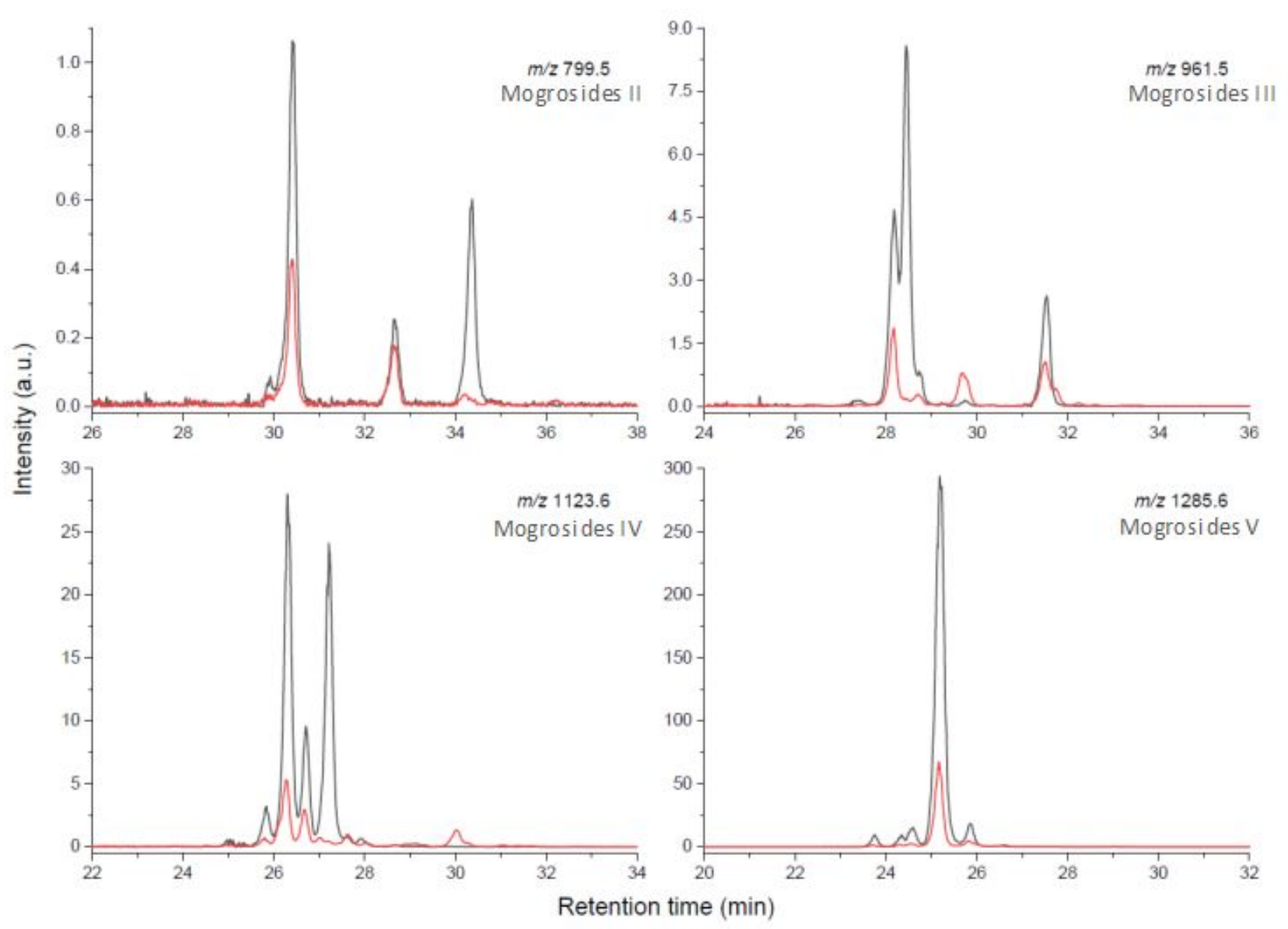

Figure 5. 


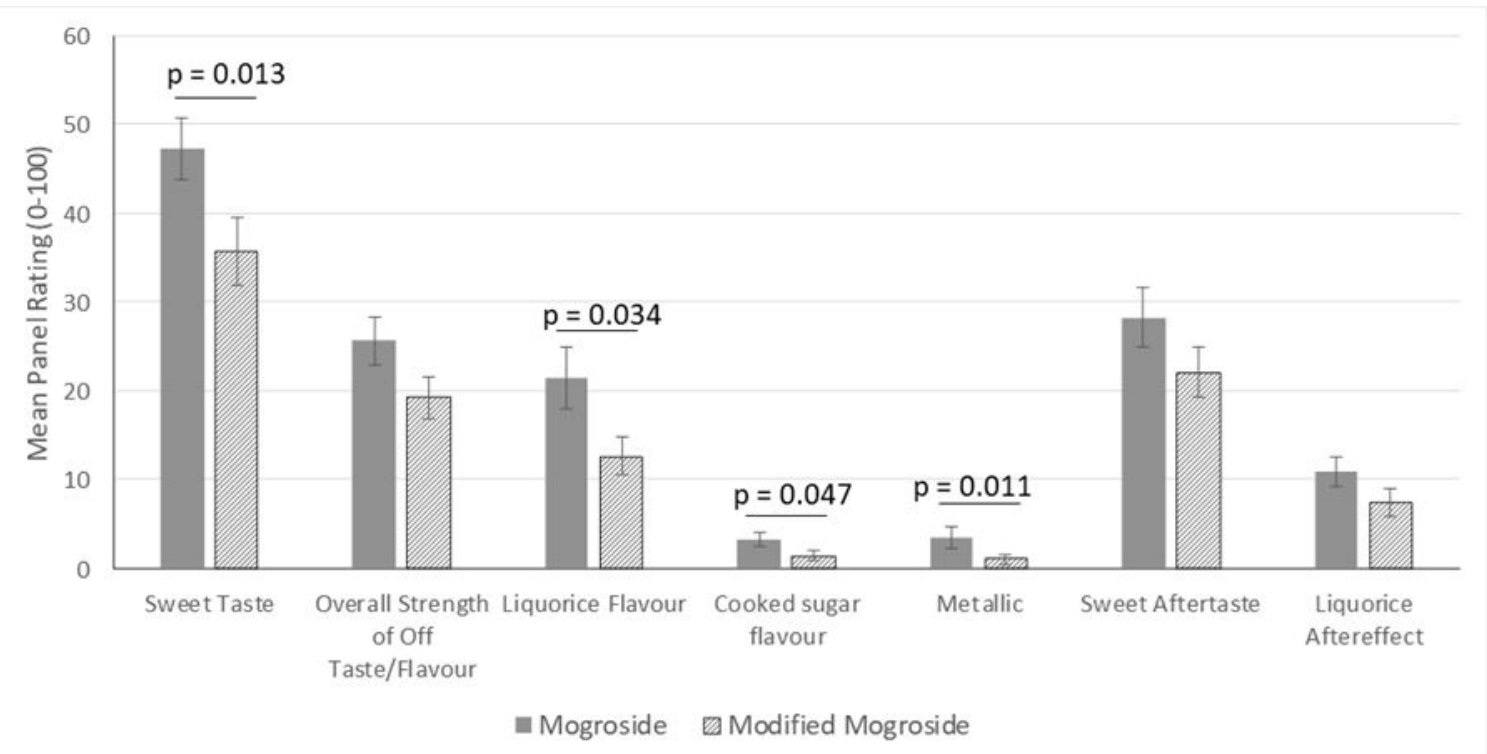

Figure 6. 
Table 1. Experimental design by using a fractional factorial design $2^{(6-2)}$ and the corresponding responses per enzyme.

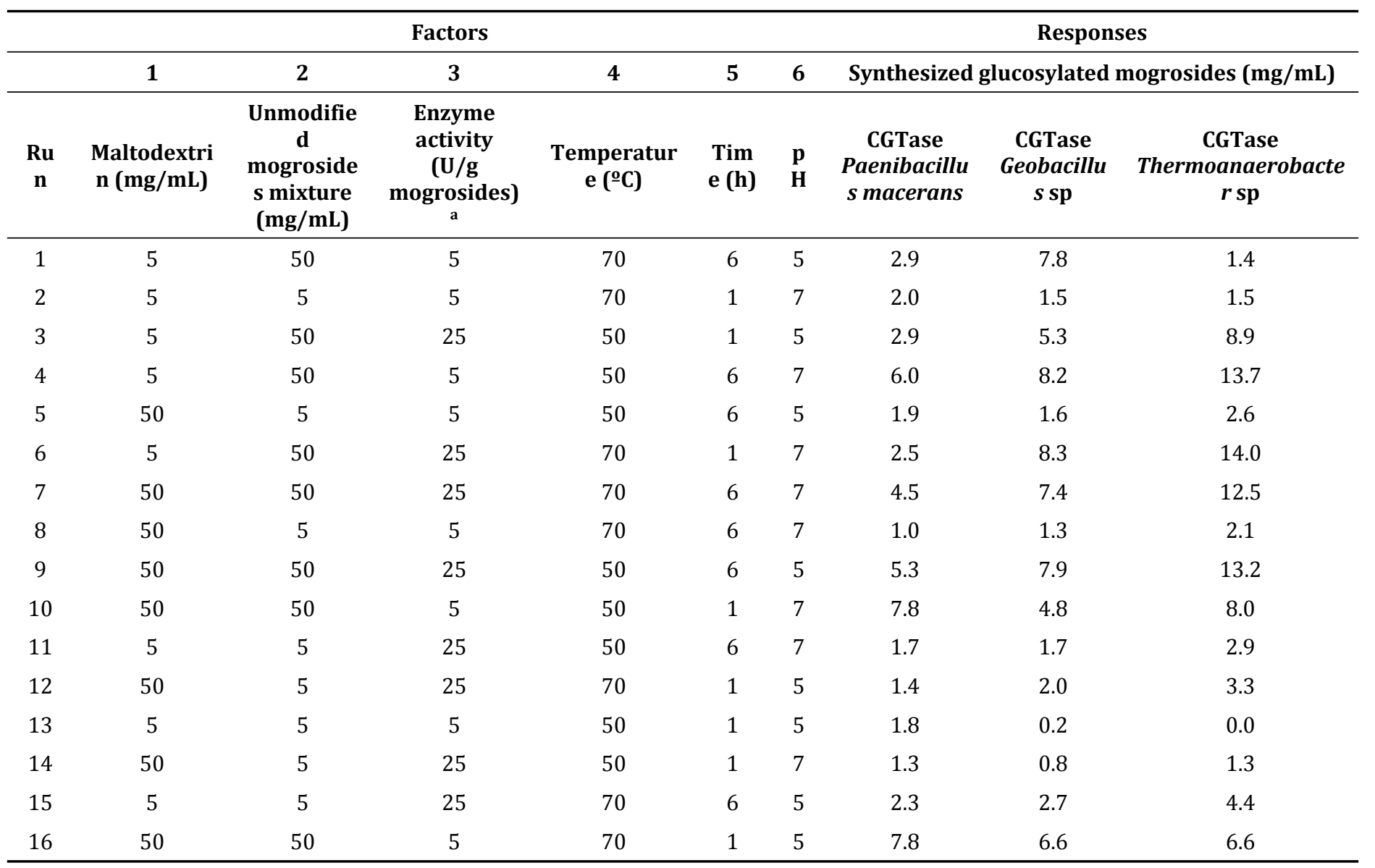

${ }^{a}$ Enzyme activity (U) was declared by the supplier and determined by methods described elsewhere based on cyclization activity ${ }^{26}$ 
Table 2. Experimental value obtained from the model equation 1 by maximizing the response.

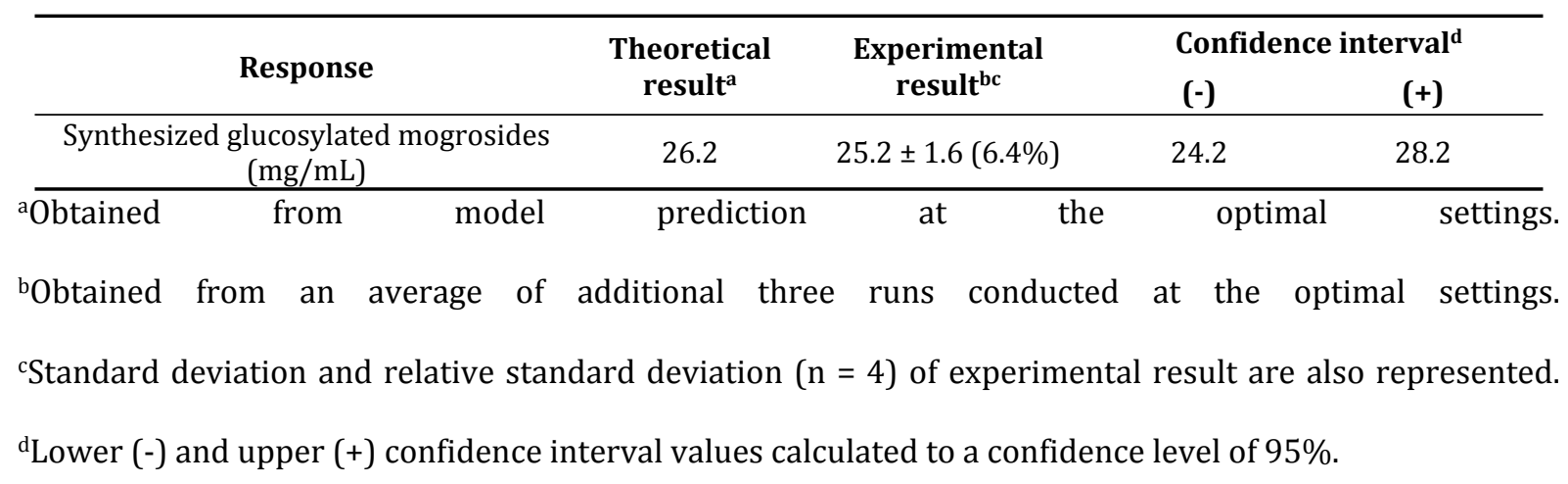


Table 3. Equivalent sucrose and potency for unmodified and modified mogrosides by CGTase from Thermoanaerobacter sp.

\begin{tabular}{ccc}
\hline & $\begin{array}{c}\text { Equivalent } \\
\text { sucrose (\%) }\end{array}$ & Potency \\
\hline Mogrosides & 4.5 & 188 \\
Modified mogrosides & 3.7 & 156 \\
\hline
\end{tabular}


TOC GRAPHIC

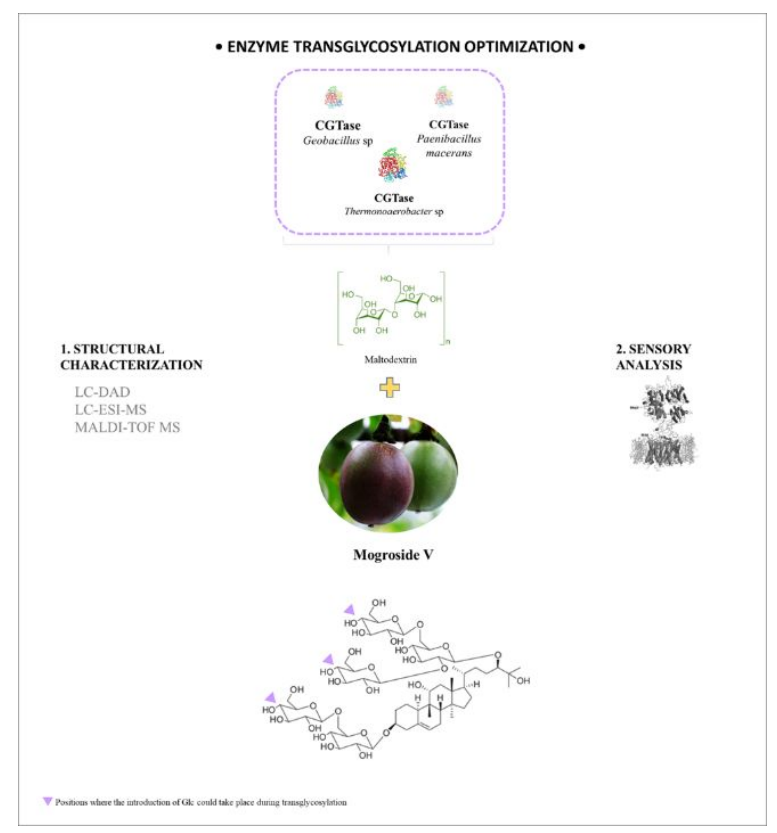

